\title{
BMJ open Strategies to increase influenza vaccination rates: outcomes of a nationwide cross-sectional survey of
UK general practice
}

\author{
Laura J Dexter, ${ }^{1}$ M Dawn Teare, ${ }^{2}$ Matthew Dexter, ${ }^{3}$ A Niroshan Siriwardena, ${ }^{4}$ \\ Robert C Read ${ }^{1}$
}

To cite: Dexter LJ, Teare MD, Dexter M, et al. Strategies to increase influenza vaccination rates: outcomes of a nationwide cross-sectional survey of UK general practice. BMJ Open 2012;2:e000851.

doi:10.1136/

bmjopen-2011-000851

- Prepublication history and additional tables for this paper are available online. To view these files please visit the journal online (http://dx. doi.org/10.1136/ bmjopen-2011-000851).

Received 6 January 2012 Accepted 3 April 2012

This final article is available for use under the terms of the Creative Commons Attribution Non-Commercial 2.0 Licence; see http://bmjopen.bmj.com

For numbered affiliations see end of article.

Correspondence to

Laura J Dexter;

I.dexter@sheffield.ac.uk

\section{ABSTRACT}

Objective: To identify practice strategies associated with higher flu vaccination rates in primary care.

Design: Logistic regression analysis of data from a cross-sectional online questionnaire.

Setting: 795 general practices across England. Participants: 569 practice managers, 335 nursing staff and 107 general practitioners.

Primary outcome measures: Flu vaccination rates achieved by each practice in different groups of at-risk patients.

Results: 7 independent factors associated with higher vaccine uptake were identified. Having a lead staff member for planning the flu campaign and producing a written report of practice performance predicted an $8 \%$ higher vaccination rate for at-risk patients aged $<65$ years (OR $1.37,95 \% \mathrm{Cl} 1.10$ to 1.71). These strategies, plus sending a personal invitation to all eligible patients and only stopping vaccination when Quality and Outcomes Framework targets are reached, predicted a $7 \%$ higher vaccination rate (OR $1.45,95 \% \mathrm{Cl} 1.10$ to 1.92$)$ in patients aged $\geq 65$ years. Using a lead member of staff for identifying eligible patients, with either a modified manufacturer's or in-house search programme for interrogating the practice IT system, independently predicted a $4 \%$ higher vaccination rate in patients aged $\geq 65$ years (OR 1.22, $95 \% \mathrm{Cl} 1.06$ to $1.41 / \mathrm{OR} 1.20$, $95 \% \mathrm{Cl} 1.03$ to 1.40 ). The provision of flu vaccine by midwives was associated with a $4 \%$ higher vaccination rate in pregnant women (OR 1.19, 95\% Cl 1.02 to 1.40$)$.

Conclusions: Clear leadership, effective communication about performance and methods used to identify and contact eligible patients were independently associated with significantly higher rates of flu vaccination. Financial targets appear to incentivise practices to work harder to maximise seasonal influenza vaccine uptake. The strategies identified here could help primary care providers to substantially increase their seasonal flu vaccination rates towards or even above the Chief Medical Officer's targets.

\section{ARTICLE SUMMARY}

Article focus

- Uptake of seasonal influenza vaccination in the UK's at-risk population is below the national and international target of $75 \%$.

- Evidence-based guidance, to advise practices how to optimise all aspects of their flu vaccination campaigns and maximise their likelihood of protecting at-risk patients against flu and its serious sequelae, is greatly needed.

- This study sought to identify which strategies and procedures were associated with higher rates of flu vaccine uptake.

Key messages

- This study has identified seven key strategies that were significantly associated with the success of practices' seasonal flu vaccination campaigns.

- If widely implemented by general practices, average vaccination rates would be predicted to rise by $7 \%-8 \%$ (thereby exceeding WHO target in patients aged $>65$ years).

Strengths and limitations of this study

- The study sample was large and representative, despite a participation rate of only $27.5 \%$.

- Outcome measures (vaccination rates) were objective and corrected for practice size.

- Strategies used to provide and encourage vaccination were self-reported.

\section{INTRODUCTION}

Influenza

Influenza (flu) is a common potentially severe but preventable infection that places a high burden on patients and healthcare providers. ${ }^{12}$ A safe and effective inactivated (killed) vaccine is produced ahead of each flu season, based on strain recommendations provided by WHO and is offered to at-risk groups in the UK free of charge. ${ }^{3-6}$ These groups have been chosen based on evidence showing increased risk of severe flu infection 
or sequelae: epidemiological data from 2010 to 2011 indicated that patients in a risk group due to chronic disease had a 10-fold greater risk of mortality due to influenza compared with those who were not in an atrisk group. ${ }^{1}$ During the 2010-2011 flu season, 602 deaths in the UK were due directly to influenza. ${ }^{1}$ Of those who died, approximately two-thirds were in a clinical risk group that is targeted for vaccination, while only $25 \%$ had received vaccination for that season.

WHO guidance indicates that developed countries should achieve $75 \%$ influenza vaccine coverage in older people, while the European Union Council (EC) advises that members should aim to vaccinate $75 \%$ of all those at high risk from influenza infection. ${ }^{7} 8$ England's Chief Medical Officer (CMO) has instructed that, in 2011-2012, each practice should aim to reach or exceed $75 \%$ uptake for people aged 65 years or over and $60 \%$ uptake for at-risk people under age 65 (increasing to $75 \%$ by $2013 / 2014) .{ }^{6}$ Published data suggest that approximately 27\% of England's population was eligible for free flu vaccination in 2010. ${ }^{9} 10$ Providing seasonal influenza vaccination is a large and complex task, which is performed well in the UK, in comparison to many other European countries. ${ }^{11}$ Over 10 million patients were vaccinated in England in the 2010-2011 season: each general practice vaccinating an average of approximately 1000 patients, mostly within a period of $4-6$ weeks. The proportion of people aged over 65 years in England who received the $2010 / 2011$ influenza vaccine was, at $72.8 \%$, just below the target of $75 \%{ }^{9}$ However, both past and current rates of vaccination in the under 65 at-risk groups fall far short of the EC or CMO targets: during 2010/ 2011, the rate achieved was 50.4\%; in pregnant women who were not otherwise at risk it was only $36.6 \%$, despite increasing evidence showing the beneficial effects of protection against flu for both mothers and babies. ${ }^{9}$ 12-14

A few previous studies have investigated the utility of specific interventions (such as telephone calls or letters) to generate an increase in uptake, but an optimal overall strategy for primary care providers remains undefined. ${ }^{15}{ }^{16}$ In this study, we aimed to investigate the entire process of flu vaccine provision in a wide range of UK general practices in order to determine the correlates of higher vaccine uptake and to inform comprehensive evidence-based recommendations for best practice.

\section{METHODS}

\section{Survey development}

Individual or small-group interviews with general practitioners (GPs), nurses and practice managers from six practices already achieving high rates of flu vaccination in urban (city), semi-rural (market town) and rural (village) areas were carried out, during which staff were asked to identify the factors considered by practices in designing and carrying out their flu vaccination campaigns. The information gained was used to construct three online questionnaires (one each for the participating groups of GPs, practice managers and practice nurses), using the Survey Monkey web-based software. ${ }^{17}$ The format of the questions and the layout of the questionnaires were designed to optimise the statistical utility of the data to be collected. The questionnaires were piloted in the same six practices to further ascertain relevance and usability before final distribution.

\section{Questionnaire distribution and survey participants}

We aimed to distribute the questionnaires to all registered GPs, practice nursing staff and practice managers within four Strategic Health Authorities (SHAs) (East Midlands, London, West Midlands and Yorkshire and Humber), which together provide care for approximately $40 \%$ of the UK population. Details about the survey were sent via the Public Health teams in the participating SHAs, who were requested to cascade the information to members of individual primary care teams (GPs, nurses and practice managers) in their area via normal electronic information circulation mechanisms. The same method was used for all other communications with participants during the study. A preliminary email, containing a letter informing primary care teams about the forthcoming study, was distributed 2 weeks before the first survey invitation. Two emails, inviting participation in the survey and containing web links to the online questionnaires, were then distributed in two consecutive weeks during August 2011.

\section{Vaccine uptake data and other comparators}

The Immform web service, which is a UK Department of Health website for collecting vaccination data from general practices, was used to obtain practice-level flu vaccination uptake data for the period 1 September 2010 to 28 February $2011 .^{18}$ Actual numbers vaccinated and actual numbers eligible were recorded for $65+$-year-olds and at-risk $<65$-year-olds (including pregnant women). These are standard groups used for targeting and measuring influenza vaccine uptake by WHO. Total practice population size was also recorded. Other practice-level data, including summary Quality and Outcomes Framework (QOF) scores (most recent data available for April 2009 to March 2010) and a variety of demographic measures, were obtained in order to identify and/or adjust for other factors which may differentially affect vaccine uptake rates (such as overall practice quality performance, practice size, population ethnicity or population deprivation). ${ }^{19}{ }^{20}$ QOF is a programme of annual financial rewards for GP surgeries, which forms part of the GP contract in England and Wales. ${ }^{21}$ The above information was linked to the questionnaire responses by NHS practice code (a unique six-figure identifier); codes were then removed from the data set prior to analysis.

\section{Statistical analyses}

In addition to a full descriptive analysis of the variables recorded in the questionnaires, logistic regression analyses were performed using STATA to compare the proportions of patients vaccinated across different 
categorical responses in the survey questionnaire. The logistic regression was based upon absolute numbers of patients vaccinated out of the total at-risk to account for differences in at-risk practice population size. Potentially confounding variables, such as total practice size, Index of Multiple Deprivation and proportion white ethnicity, were analysed for correlation with vaccination uptake and adjusted for in the univariable analyses where appropriate. As there was not always complete agreement in questionnaire responses received from different participants in the same practice, robust standard errors were computed using a cluster correction model, thereby generating data corrected to the practice level. Multivariable regression analysis was then performed on any two or more results from the same subset(s) of participants (ie, practice managers, nurses and/or GPs), which showed significance at the $95 \%$ level on univariable analysis.

\section{RESULTS}

Fifty primary care trusts distributed the survey invitation to a total of 2896 practices. Responses were submitted by 569 practice managers, 335 nursing staff and 107 GPs, representing 795 practices $(27.5 \%$ of those invited to contribute). These practices serve a total of approximately 5.8 million patients, among whom over 1.5 million are eligible for influenza vaccination. The distributions of flu vaccination rates in the surveyed practices were well matched to national patterns (figure 1). For the majority of eligible patients (ie, those aged 65 years and above), our findings indicated that the variation between practices' flu vaccination rates was not influenced by differences in the ethnicity or affluence of their patient populations, whereas Quality and Outcomes Framework summary scores showed highly and significant positive correlations with vaccine uptake

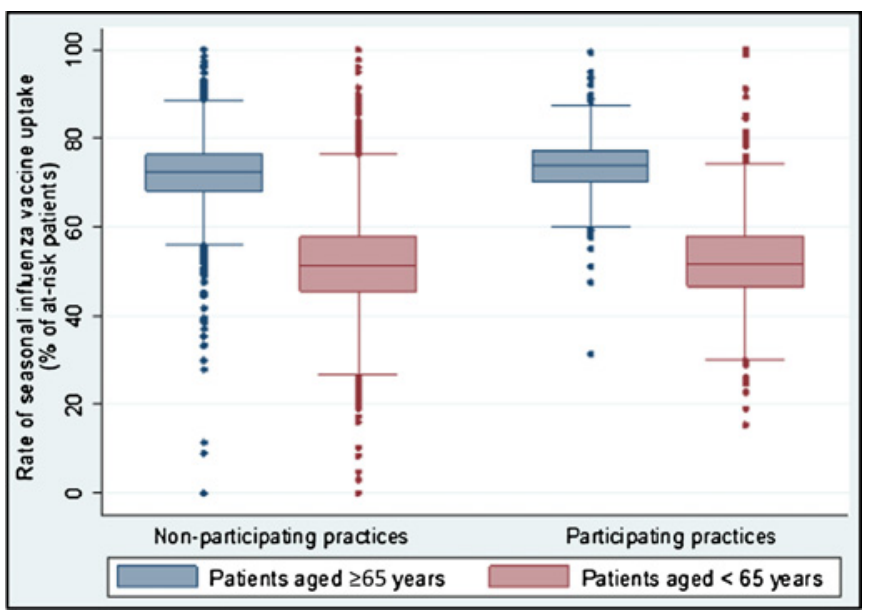

Figure 1 Box and whisker plots showing the range and distribution of influenza vaccination uptake rates for patients aged 65+ years (blue boxes and bars) and at-risk patients aged under 65 years (red boxes and bars). The distribution of uptake rates for non-participating practices $(\mathrm{N}=2101)$ and participating practices $(\mathrm{N}=795)$ are not significantly different. achieved in both age groups (supplementary table 1). The univariable logistic regression results referred to throughout this section are shown in supplementary table 2 .

\section{Staffing}

Having a lead member of staff for arranging the practice flu vaccination campaign was associated with increased flu vaccine uptake rates in both $65+$ and $<65$ age groups $(\mathrm{p}=0.001$ and 0.004 , respectively). Nominating a staff member with responsibility for identifying eligible patients was associated with increased uptake of vaccine in older age groups $(\mathrm{p}=0.038)$, but this trend, although present, did not reach significance in under 65s $(\mathrm{p}=0.218)$.

\section{Ordering vaccine}

A plot of the number of vaccine doses ordered for 2011-2012 versus vaccines used in 2010-2011 shows a tight correlation (figure 2A). On average, practices were found to have ordered vaccines based on the number of doses given in the previous season, with an average uplift of $8.8 \%$ (95\% CI $4.3 \%$ to $13.3 \%$; $n=568$ ). As vaccines are ordered as a total, these data could not be differentiated into doses intended for vaccination of patients aged over or under 65 years old. However, the data showed that only $78.3 \%$ of responding practices would have been able to vaccinate at least $75 \%$ of their at-risk patients (in accordance with the CMO recommendations) (figure $2 \mathrm{~B}$ ).

\section{Contacting patients}

Using personal invitations, either alone or in combination with general publicity, was significantly associated with higher rates of vaccination. The use of personal invitations for all patients (not just those who did not respond to an initial general publicity campaign) was associated with the highest vaccination rates in the larger $65+$ age group $(p=0.003)$, although a similar association did not reach statistical significance in the under 65 s. Using both letters and telephone calls was not associated with significantly different vaccination rates than using either letters or phone calls alone $(p=0.721$ for patients aged $65+; \mathrm{p}=0.852$ for patients aged $<65)$.

\section{Identifying eligible patients}

Programmes for identifying eligible patients from the practices' IT system are usually issued by the software providers. Modifying the IT supplier's standard search or creating a separate in-house search was associated with significantly higher uptake rates for patients aged 65 + years than using an unmodified IT supplier's search $(\mathrm{p}<0.001$ and 0.027 , respectively). A similar trend for under $65 \mathrm{~s}$ did not reach statistical significance, perhaps due to insufficient power. As older patients are identified simply on the basis of their age at a certain date, which should not require a complicated search strategy, these findings suggest that creating or modifying a system search reflects that the staff in these practices are more 
Figure 2 (A) Relationship between reported total number of vaccine doses ordered for the 2011-2012 season and actual number of doses administered in 2010-2011 $(\mathrm{n}=568)$ and (B) plot showing the maximum average achievable vaccination rates for the 2011-2012 season, based on the total number of vaccine doses ordered and the total number of eligible patients. Red line indicates the Chief Medical Officer's target of $75 \%$.
A

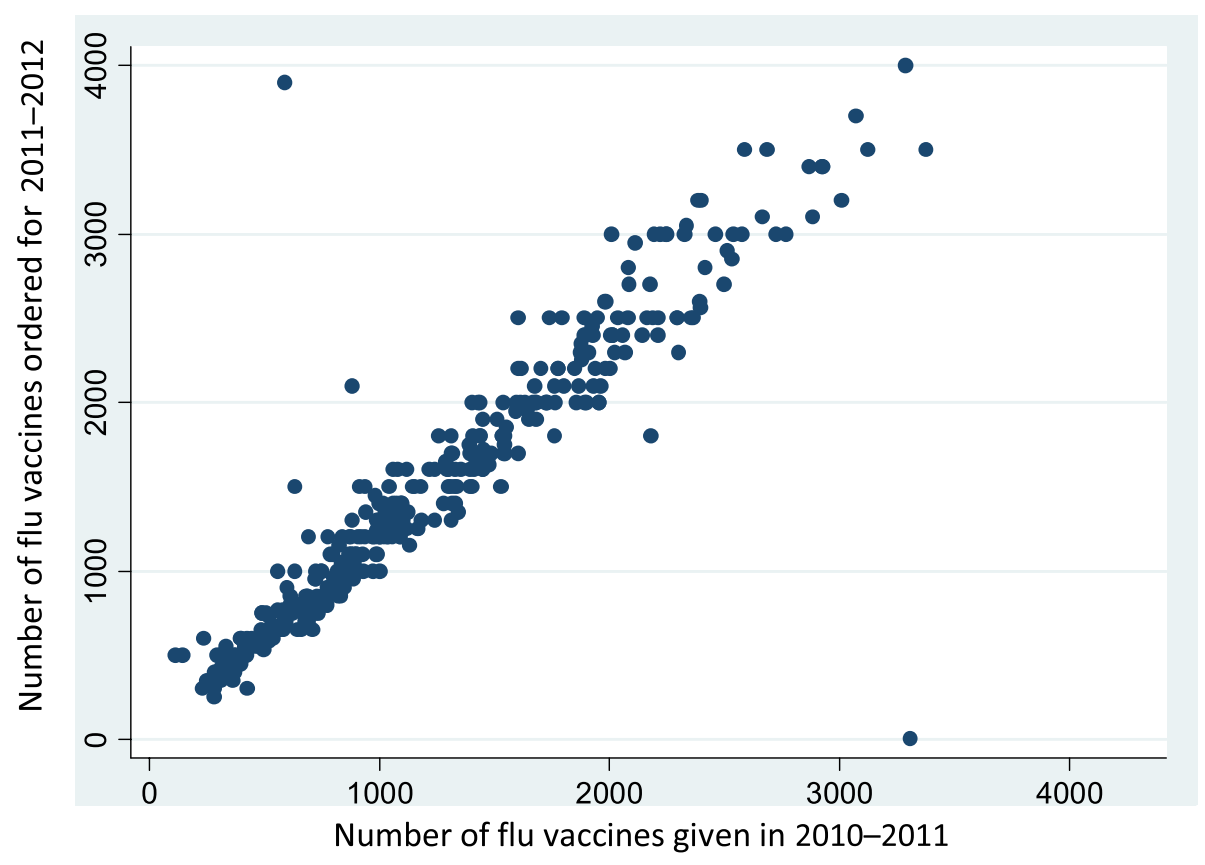

B

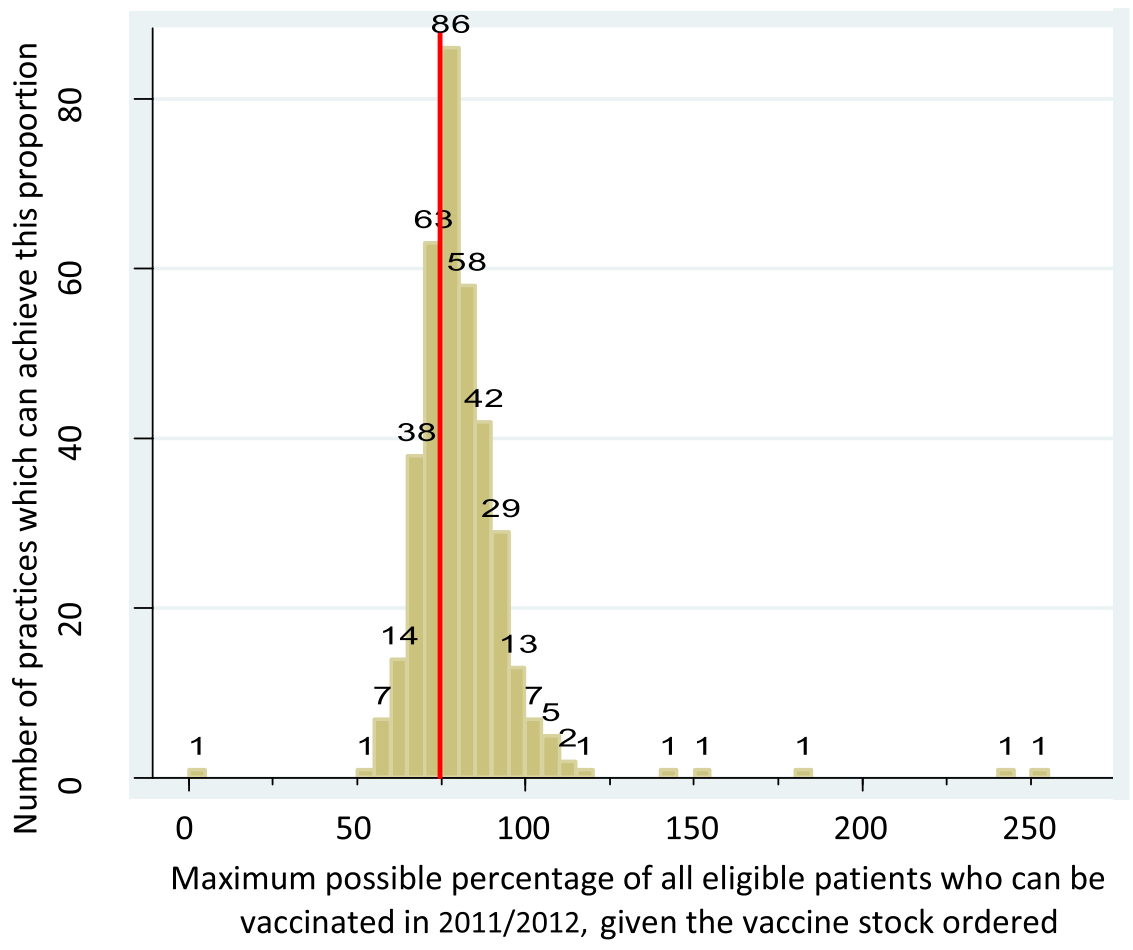

motivated and/or experienced to use their IT system to try to achieve their flu vaccination targets.

\section{Offering clinics and appointments}

More than $95 \%$ of practices held the main vaccination sessions at their usual surgery premises and $75 \%$ held the main sessions during normal surgery hours. Most practices reported using a variety of appointment types and timings to provide flu vaccination. Surprisingly, in our data, offering vaccinations at weekends, or before 8:00 or after 18:00, was not associated with a significant difference in the vaccination uptake rates achieved.
Increasing numbers of reminders or repeat invitations were associated with significantly increased vaccine uptake in the under 65-year-olds $(\mathrm{p}=0.038)$, though not vaccination (for under 65s) occurred in practices that identified appointments for flu vaccination using a specific Read (computer identification) code $(\mathrm{p}=0.038)$.

\section{Vaccinating pregnant women}

The proportion of practices that reported that their community midwives recommended flu vaccination to in those aged $65+$ years. Significantly, higher rates of 
pregnant women was disappointingly low (57.5\%). Furthermore, there was a clear discrepancy between this figure and the proportion that reported that their community midwives actually administered vaccine (17.8\%; see figure $3 \mathrm{~A})$. Our analysis demonstrated that practices where community midwives were active in administering flu vaccinations to pregnant patients achieved significantly higher rates of uptake in this particular at-risk group $(\mathrm{p}=0.023)$.

\section{Ending and reviewing the campaign}

A total of 578 practices provided information on what influenced their decision to stop offering flu vaccination. Of major concern was the evidence that almost $50 \%$ of practices stopped offering flu vaccines partly, or solely, because they had exhausted their stock. Almost one-third (28.9\%) cited a financial factor in making their decision and the data showed that ending flu vaccination only once QOF targets had been reached was associated with increased uptake rates for those aged $65+$ years $(p=0.048)$; in those aged under 65 years, this was only weakly significant $(p=0.100)$, perhaps influenced by the smaller numbers of patients in this group. These results suggest that practices that focused on financial targets were motivated and/or organised to continue their efforts to vaccinate patients beyond the point at which other practices may stop. In support of this hypothesis, we found that patients whose vaccination would contribute to a QOF-related payment received an average of $42 \%$ (95\% CI $33 \%$ to $51 \%$ ) more reminders more than those who did not have a QOF-registered indication for vaccination $(\mathrm{p}<0.001)$.

Practices which produced a written report reviewing their flu vaccination rates achieved very significantly higher vaccination rates in both younger and older age groups compared with those practices which did not produce a written report $(\mathrm{p}=0.006$ for patients aged 65 + years; $p=0.002$ for patients aged $<65$ years). Similarly, reviewing the practice's flu vaccination strategy in a written format was also significantly associated with achieving higher rates of vaccination $(p=0.067$ for patients aged $65+$ years; $\mathrm{p}=0.028$ for patients aged $<65$ years). This finding suggests that practices that produced written reports may have been able to organise more rigorous campaigns and/or have had more wellinformed and motivated staff, resulting in more effective performance.

\section{Personal motivations and attitudes of staff}

Figure 3B shows a summary of GPs', nurses' and practice managers' views of the flu vaccination campaign. There was a significant association between encouraging vaccination among colleagues and other staff and achieving higher rates of vaccine uptake in patients aged 65 years or above $(p=0.004)$ but not in those aged under 65 years $(p=0.208)$. There was a trend for a similar association between positive attitudes of staff towards being vaccinated themselves and higher rates of patient vaccination in a practice, but this did not reach statistical significance in either the older or younger age group $(\mathrm{p}=0.440$ and 0.185 , respectively).

\section{Predicted impact of strategies to increase rates of influenza vaccination}

Seven factors were found to have significant independent positive associations with flu vaccine uptake levels following multivariable regression analysis (tables 1 and 2). For patients aged $<65$ years, having a lead member of staff for planning the flu campaign and producing a written report of the practice's performance were associated with a combined OR of 1.37 , which predicts an $8 \%$ higher flu vaccination rate for practices that employ these strategies compared with those that do not $(54 \%$ vs $46 \%)$.

In patients aged 65 years or over, a further two factors were also found to remain independently correlated with increased rates of flu vaccination. These were sending a personal invitation to all eligible patients and
Figure 3 Showing $(A)$ the reported activity of community midwifery teams in recommending and providing seasonal influenza vaccination to pregnant women and $(B)$ the attitudes of participating healthcare workers to vaccination of colleagues and themselves.
A

My local midwives are active in recommending vaccination to pregnant patients

My local midwives are active in providing vaccination to pregnant patients

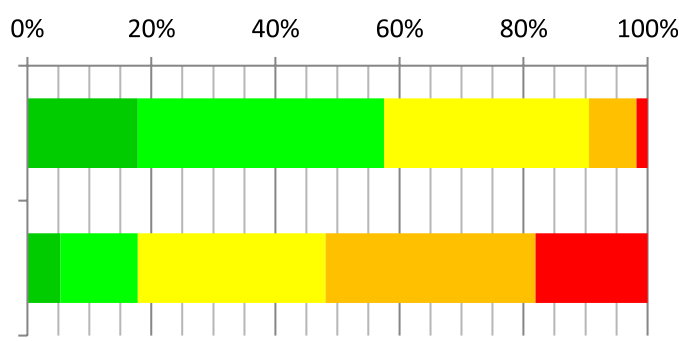

B

I encourage my staff/colleagues to receive vaccination

As a healthcare worker, I am happy to have the flu vaccine myself

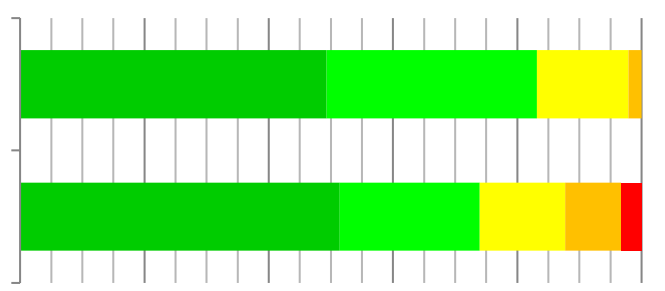


Table 1 Statistically significant factors found on multivariate regression analysis of responses common to all three types of staff (general practitioners, nursing staff and practice managers)

\begin{tabular}{|c|c|c|c|c|}
\hline Description of significant factor & $\begin{array}{l}\text { Regression } \\
\text { co-efficient }\end{array}$ & $95 \% \mathrm{Cl}$ & p Value & $\begin{array}{l}\text { Number } \\
\text { of clusters }\end{array}$ \\
\hline \multicolumn{5}{|l|}{ For patients aged $\geq 65$ years } \\
\hline Producing a written report to review flu vaccine uptake rates & 0.065 & 0.023 to 0.107 & 0.010 & 659 \\
\hline $\begin{array}{l}\text { Having a lead member of staff for planning the practice's } \\
\text { flu vaccination campaign }\end{array}$ & 0.144 & 0.035 to 0.253 & 0.010 & 659 \\
\hline Sending a personal invitation to all eligible patients & 0.081 & 0.035 to 0.127 & 0.001 & 659 \\
\hline $\begin{array}{l}\text { Only stopping vaccination when Quality and Outcomes } \\
\text { Framework targets are reached }\end{array}$ & 0.085 & 0.004 to 0.166 & 0.039 & 659 \\
\hline \multicolumn{5}{|l|}{ For at-risk patients aged $<65$ years } \\
\hline Producing a written report to review flu vaccine uptake rates & 0.113 & 0.042 to 0.184 & 0.002 & 783 \\
\hline $\begin{array}{l}\text { Having a lead member of staff for planning the practice's } \\
\text { flu vaccination campaign }\end{array}$ & 0.203 & 0.054 to 0.352 & 0.008 & 783 \\
\hline
\end{tabular}

only stopping vaccination when QOF targets are reached. The overall OR associated with the implementation of all four strategies in this age group was 1.45 , which predicts a $7 \%$ higher vaccination rate in this age group when these strategies are used (78\% vs $71 \%)$.

The strategies of using a lead member of staff for identifying eligible patients and either a modified manufacturer's search programme or an in-house search programme for interrogating the practice IT system were also independently correlated with increased rates of flu vaccination in patients aged 65 years or more. However, the effect seen was weaker as these data are derived from a subset of responses from practice managers only, suggesting a rise to $78 \%$ from a baseline of $74 \%$.

The active involvement of midwives in providing flu vaccination was significantly associated with higher levels of vaccine uptake in pregnant women but, as the only significant variable within this group, the finding could not be included in a multivariable analysis. However, applying the OR of 1.20 predicted by the univariable analysis, our data indicate that the provision of vaccination by midwives rather than GPs is associated with an increase in uptake rate to $45 \%$ in pregnant women (from an observed average baseline vaccination rate of $41 \%$ in our cohort).

\section{DISCUSSION}

This study has identified seven factors which GPs might use to improve and maximise uptake of seasonal flu vaccine in at-risk patients. Many of these strategies are common sense and align with the empirical guidance given by the English and Scottish CMOs, ${ }^{22}{ }^{23}$ but our study provides the first statistical evidence to support the validity of such approaches. The study sample was large and appeared to be representative of the overall cohort, despite a participation rate of only $27.5 \%$. Although we found no evidence for selection bias of participating practice staff, when the vaccination rates of participating practices were compared with national data, it remains possible that the responses of staff who completed the questionnaire did not reliably represent the views of all practice staff within those participating practices. However, most of our questions sought factual data rather than opinions and so this bias, if present, is likely to be limited.

This study focused on identifying strategies and approaches that GPs might use or influence to improve vaccine uptake. However, numerous patient-related factors also affect flu vaccine uptake. Highly mobile or ethnically diverse populations may prove very difficult to contact and target for flu vaccination. It is also likely that a minority of patients will always refuse or miss vaccination, no matter how much GPs strive to provide it. Public perception of influenza as a significant threat to health and of vaccination as an effective preventive strategy is associated with higher uptake. ${ }^{24}$ People who receive information about these factors from official health sources (particularly GPs or nurses in the primary care

Table 2 Statistically significant factors found on multivariate regression analysis of responses from practice managers only

\begin{tabular}{|c|c|c|c|c|}
\hline Description of significant factor & $\begin{array}{l}\text { Regression } \\
\text { co-efficient }\end{array}$ & $95 \% \mathrm{Cl}$ & p Value & $\begin{array}{l}\text { Number } \\
\text { of clusters }\end{array}$ \\
\hline \multicolumn{5}{|l|}{ For patients aged $\geq 65$ years } \\
\hline $\begin{array}{l}\text { Identifying eligible patients using a modified manufacturer's } \\
\text { search programme }\end{array}$ & 0.115 & 0.056 to 0.175 & 0.000 & 395 \\
\hline Identifying eligible patients using an in-house search programme & 0.096 & 0.028 to 0.163 & 0.006 & 395 \\
\hline $\begin{array}{l}\text { Having a lead member of staff for identifying eligible patients } \\
\text { in the practice }\end{array}$ & 0.086 & 0.001 to 0.170 & 0.046 & 395 \\
\hline
\end{tabular}


setting) and who think that others want them to be vaccinated are more likely to get vaccinated. ${ }^{1124} 25$ In line with this, flu vaccination uptake is greater among older people and others who make routine use of hospital and community care services. ${ }^{26}$ Unsurprisingly, fear of side effects of vaccination is a strong negative influence, while lack of general motivation and ignorance about the recommendations are other commonly reported barriers to both seasonal and pandemic vaccination. ${ }^{27}{ }^{28}$ However, when intensive recall stimuli and information are provided to at-risk patients, as few as $3.5 \%$ will refuse vaccination, suggesting that patient attitudes are malleable and should not present a barrier to achieving the CMO's aims. ${ }^{29}$ Our results strongly support the provision of personal invitations for all patients (in alternative languages and/or formats, if required), as advised by the CMO. ${ }^{22}$ However, we found little evidence of benefit from offering very early (before 8:00), late (after 18:00) or weekend appointments. This contradicts some current guidance ${ }^{22}$ and is likely to be of financially relevance for practices. Our findings with respect to the flu vaccination of pregnant women are also important. Although administering flu vaccine is not part of the current role of many midwives, it is logical that the ability both to discuss and to provide vaccination to pregnant women would increase uptake in this risk group by removing the need for referral and attendance at a separate clinic: our analyses now support this logic. However, if midwives were to provide influenza vaccination outside of the practice setting, it would be essential for reliable records of this to be transferred to the GP.

The need for good communication with patients, to encourage the uptake of flu vaccination, is axiomatic. However, several statistically significant outcomes of our analyses have not previously been described and are directly or indirectly associated with the quality or extent of communication within practices. The production of a written review of practice performance might be associated with higher flu vaccination rates for a number of reasons. Production of such a report indicates that at least one member of staff must be able to access and manipulate the relevant data using their practice's computer systems and is motivated to do so. Subsequent dissemination of the report allows staff to become aware of their practice's performance and identify areas for improvement. Only $20 \%$ of practices in our study produced a written report of vaccine uptake rates and this strategy is not currently recommended in the CMO guidance for England. Our results also indicate that each practice should nominate lead members of staff for organising the practice's influenza vaccination campaign (as advised by the English and Scottish CMOs ${ }^{22} 23$ ) and for identifying at-risk patients from the practice database. This is supported by a recently published study from the USA, which suggested that effective use of electronic databases by a skilled data manager could increase the rate of flu vaccination by over $10 \% .^{30}$ The ability to perform a modified or in-house search of the practice's patient database is unlikely to improve the simple process of identification of those aged over 65 years. However, a member of staff who is thoroughly familiar with the IT system may be more able to contribute to improved rates of vaccination by, for example, monitoring levels of appointment bookings and vaccine uptake in real-time throughout the campaign; generating automatic invitation and reminder letters, text messages or emails; creating alert flags on the patient record to promote opportunist vaccination. Our study's findings also suggest that the effectiveness of a practice's flu vaccination campaign is increased when staff promote vaccination among themselves, an effect which may arise from increased motivation for the campaign as a whole or through communication of their positive attitude to at-risk patients. This supports the findings of previous studies and should thus contribute to an increased impetus to encourage vaccination of staff. ${ }^{31} 32$

The relationship between staff motivation and practice performance is neither simple nor exclusive. Individuals may have different motivations and their interaction within a larger team may produce variable outcomes. We do not argue that introducing written reports and/or tailoring the practice search strategies would necessarily increase motivation. However, in practices where these actions are not already undertaken, their introduction would ensure that staff become more aware of the practice's performance and the underlying mechanisms that influence it. Having found a highly significant correlation of these strategies with increased vaccine uptake, we propose that increased awareness and knowledge may help to increase staff motivation. However, our study was not designed to measure motivation per se or its effects in isolation and this would be an interesting (though challenging) area for further study.

There is currently a significant financial risk for practices attempting to improve their vaccination rates. As practices are only reimbursed on the basis of the number of vaccines administered, they face a financial penalty if they buy more doses than are used and sale-or-return schemes are usually limited to a few per cent of the vaccine doses in the overall order. Perhaps as a result, we have found that almost $50 \%$ of practices currently halt their vaccination campaigns due to exhaustion of vaccine stocks. A central procurement strategy for flu vaccines, which has recently undergone consultation by the Department of Health, should remove this financial stricture and allow practices to aim for much higher vaccination rates without risking financial penalty. ${ }^{33}$ However, this will also result in the loss of a significant proportion of practices' funding for flu vaccination (ie, which is currently derived from any discrepancy between tariff price and purchased price for the vaccine itself). Considerable effort and resources are required to deliver a successful flu vaccination campaign, and our findings indicate that practices' efforts can be influenced by 
financial motivations. The pursuit of QOF targets for flu vaccination requires practice staff to be able to perform complex interrogations of their patient database and to be aware of rates of vaccine uptake while the flu vaccination campaign is progressing. Our data suggest that pursuing the QOF targets may motivate practices to maintain vaccine stocks and encourage extra patients to receive vaccine. We would not advise that practices should automatically stop vaccinating patients once their QOF targets have been attained. However, our findings suggest that a scale of financially supported targets applicable across all patient groups, or the inclusion of flu vaccination of all at-risk patients in the QOF scheme, might be a powerful tool to increase flu vaccine uptake.

Current vaccines achieve around 50\%-80\% protection against influenza and associated sequelae in at-risk groups. ${ }^{3}{ }^{34-36}$ However, these efficacy rates do not translate into public health protection if the vaccine is not delivered effectively to the communities that need it. With flu vaccination rates varying from $15 \%$ to $100 \%$ (figure 1) between the worst and best practices in our nationwide cohort, there is the potential for enormous gains to be made. This study has identified seven simple steps that can improve our performance and increase the protection of at-risk patients.

\section{Author affiliations}

${ }^{1}$ Department of Infection and Immunity, The University of Sheffield Medical School, Sheffield, UK

${ }^{2}$ Health Services Research, School of Health and Related Research, University of Sheffield, Sheffield, UK

${ }^{3}$ Retford Health, Retford Primary Care Centre, Retford, UK

${ }^{4}$ Lincoln School of Health and Social Care, University of Lincoln, Brayford Pool, Lincoln, UK

Acknowledgements We are grateful to the survey participants for taking part; to Dr Mark Ashworth, GP and Clinical Senior Lecturer at King's College London for providing the demographic and deprivation data; to Dr Helen Carter, Consultant in Public Health at NHS West Midlands, Dr Andrew Clark, Deputy Director of Public Health at NHS Yorkshire and Humber, Dr Giri Rajaratnam, Deputy Regional Director of Public Health at NHS East Midlands, Dr Leslyn Rew, Project Manager at NHS West Midlands, and Dr Chloe Sellwood, Pandemic Flu Lead at NHS London and their Vaccination \& Immunisation teams, for arranging circulation of the survey details to primary care teams; to staff at Bridgegate, Crown House, Riverside, and Tall Trees Surgeries in Retford, Charnock Health Primary Care Centre in Sheffield and Bawtry and Blyth Health Centre in Bawtry for feedback on questionnaire and guideline design and to Mr Gerald Ellis, Business Manager at Retford Health, for co-ordinating feedback.

Contributors LJD designed, carried out and coordinated analysis and reporting of the study and also drafted and revised this manuscript. MDT contributed to statistical aspects of design, carried out the formal data analysis and contributed to the manuscript. MD and ANS contributed to primary care and policy aspects of study design and interpretation and also revised the manuscript. RCR contributed to study design and interpretation and revised the manuscript. He is guarantor.

Funding This study was an independent report commissioned and funded by the Policy Research Programme in the Department of Health, UK (reference number 039/0035). The views expressed are not necessarily those of the Department. The study was sponsored and granted ethical approval by the University of Sheffield. Neither the study sponsor nor the funder played any role in study design; in the collection, analysis and interpretation of data; in the writing of the report or in the decision to submit the article for publication. All authors had access to the study data (including statistical reports and tables) and can take responsibility for the integrity of the data and the accuracy of the data analysis.
Competing interests None.

Ethics approval Ethics approval was provided by the University of Sheffield.

Provenance and peer review Not commissioned; externally peer reviewed.

Data sharing statement The availability of additional data is subject to consent for its dissemination from the Department of Health Central Commissioning Facility.

\section{REFERENCES}

1. Surveillance Of Influenza And Other Respiratory Viruses In The UK 2010/11. London, UK: Health Protection Agency, 2011.

2. Fleming DM, Elliot AJ, Nguyen-Van-Tam JS, et al. A Winter's Tale: Coming To Terms With Winter Respiratory IIInesses. London, UK: Health Protection Agency, 2005.

3. Pebody R, Hardelid P, Fleming D, et al. Effectiveness of seasonal 2010/11 and pandemic influenza $A(H 1 N 1) 2009$ vaccines in preventing influenza infection in the United Kingdom: mid-season analysis 2010/11. Euro Surveill 2011;16:19791.

4. Mangtani $\mathrm{P}$, Cumberland $\mathrm{P}$, Hodgson $\mathrm{CR}$, et al. A cohort study of the effectiveness of influenza vaccine in older people, performed using the United Kingdom general practice research database. J Infect Dis 2004;190:1-10.

5. Influenza [chapter 19]. Immunisation Against Infectious Disease. UK: Department of Health, 2011.

6. Davies S. Letter from the CMO: Preparation and Assurance for 2011/12 Seasonal Influenza Immunisation Programme. UK: Department of Health, 2011.

7. 56th World Health Assembly. Prevention and Control of Influenza Pandemics and Annual Epidemics. Geneva, Switzerland: World Health Organisation, 2003.

8. Council of the European Union. Council recommendation of 22 December 2009 on seasonal influenza vaccination (2009/1019/EU). Official Journal of the European Union 2009;348:71-2.

9. Begum F, Pebody R. Seasonal Influenza Vaccine Uptake Amongst GP Patient Groups in England, Winter Season 2010-11. England: Department of Health, 2011.

10. Annual Mid-Year Population Estimates, 2010. Office for National Statistics, 2011.

11. Blank PR, Schwenkglenks M, Szucs TD. Vaccination coverage rates in eleven European countries during two consecutive influenza seasons. J Infect 2009;58:446-58

12. Puleston RL, Bugg G, Hoschler K, et al. Observational study to investigate vertically acquired passive immunity in babies of mothers vaccinated against $\mathrm{H} 1 \mathrm{~N} 1 \mathrm{v}$ during pregnancy. Health Technol Assess 2010;14:1-82.

13. Benowitz I, Esposito DB, Gracey KD, et al. Influenza vaccine given to pregnant women reduces hospitalization due to influenza in their infants. Clin Infect Dis 2010;51:1355-61.

14. Eick AA, Uyeki TM, Klimov A, et al. Maternal influenza vaccination and effect on influenza virus infection in young infants. Arch Pediatr Adolesc Med. UK, 2011;165:104-11.

15. Siriwardena AN, Rashid A, Johnson MR, et al. Cluster randomised controlled trial of an educational outreach visit to improve influenza and pneumococcal immunisation rates in primary care. $\mathrm{Br} \mathrm{J} \mathrm{Gen}$ Pract 2002;52:735-40.

16. Furey A, Robinson E, Young Y. Improving influenza immunisation coverage in 2000-2001: a baseline survey, review of the evidence and sharing of best practice. Commun Dis Public Health 2001;4:183-7.

17. http://www.surveymonkey.com (accessed 22 Jul 2011)

18. www.immform.dh.gov.uk (accessed 3 Jul 2011).

19. Practice-level ethnicity and deprivation data. $J$ Health Serv Res Policy kindly supplied by Dr Mark Ashworth, GP and Clinical Senior Lecturer, Department of Primary Care and Public Health Sciences, King's College London, UK. 2011;16:21-7.

20. http://www.ic.nhs.uk/statistics-and-data-collections/supportinginformation/audits-and-performance/the-quality-and-outcomesframework/qof-2009-10/data-tables (accessed $10 \mathrm{Jul} 2011$ ).

21. Gillam S, Siriwardena A. The Quality and Outcomes Framework: QOF Transforming General Practice. Oxford: Radcliffe Publishing Ltd, 2010.

22. Anon. Seasonal Flu Plan. UK: Department of Health, 2011.

23. Seasonal influenza and pneumococcal immunisation programmes 2010-11 (letter). Scotland: Chief medical Officer and public health Directorate, 2010

24. Bish A, Yardley L, Nicoll A, et al. Factors associated with uptake of vaccination against pandemic influenza: a systematic review. Vaccine 2011;29:6472-84.

25. Maurer J, Harris KM. Contact and communication with healthcare providers regarding influenza vaccination during the $2009-2010 \mathrm{H} 1 \mathrm{~N} 1$ pandemic. Prev Med 2011;52:459-64. 
26. Crawford VL, O'Hanlon A, McGee H. The effect of patient characteristics upon uptake of the influenza vaccination: a study comparing community-based older adults in two healthcare systems. Age Ageing 2011:40:35-41.

27. Myers LB, Goodwin R. Determinants of adults' intention to vaccinate against pandemic swine flu. BMC Public Health 2011;11:15.

28. Keenan $\mathrm{H}$, Campbell $\mathrm{J}$, Evans $\mathrm{PH}$. Influenza vaccination in patients with asthma: why is the uptake so low? Br J Gen Pract 2007;57:359-63.

29. Humiston SG, Bennett NM, Long $C$, et al. Increasing inner-city adult influenza vaccination rates: a randomized controlled trial. Public Health Rep 2011;126(Suppl 2):39-47.

30. Loo TS, Davis RB, Lipsitz LA, et al. Electronic medical record reminders and panel management to improve primary care of elderly patients. Arch Intern Med 2011;171:1552-8.

31. Abramson $\mathrm{ZH}$, Levi O. Is performance of influenza vaccination in the elderly related to treating physician's self immunization and other physician characteristics? Prev Med 2008;47:550-3.
32. Nichol KL, Zimmerman R. Generalist and subspecialist physicians knowledge, attitudes, and practices regarding influenza and pneumococcal vaccinations for elderly and other high-risk patients: a nationwide survey. Arch Intern Med 2001; 161:2702-8.

33. A Review Of The Procurement Of Seasonal Flu Vaccine 2011. UK: Immunisation Branch, Department of Health, 2011.

34. Schembri S, Morant S, Winter JH, et al. Influenza but not pneumococcal vaccination protects against all-cause mortality in patients with COPD. Thorax 2009;64:567-72.

35. Jackson LA, Gaglani MJ, Keyserling HL, et al. Safety, efficacy, and immunogenicity of an inactivated influenza vaccine in healthy adults: a randomized, placebo-controlled trial over two influenza seasons. BMC Infect Dis 2010;10:71.

36. Osterholm MT, Kelley NS, Sommer A, et al. Efficacy and effectiveness of influenza vaccines: a systematic review and meta-analysis. Lancet Infect Dis 2012;12:36-44. 